\title{
Annual and semi-annual variations of the geomagnetic field components analysed by the multi-taper method
}

\author{
I. Wardinski and M. Mandea \\ Section 2.3, Earth's Magnetic Field, Geoforschungszentrum Potsdam, Germany \\ (Received June 10, 2005; Revised February 3, 2006; Accepted February 6, 2006; Online published June 2, 2006)
}

\begin{abstract}
Monthly means of three geomagnetic components ( $X$-northern, $Y$-eastern and $Z$-vertical downward) from 154 observatories over the interval 1960-2002 have been analysed to determine the annual and semi-annual variations. The method applied is a multi-taper analysis, which gives a robust estimate of the power spectral density of the temporal variation of the field components. The main objective is to investigate the geographic dependence of the annual and semi-annual variations. Surprisingly, these variations in the vertical downward component are not homogeneously distributed all over the globe, having mainly a longitudinal dependency. The results of the analysis are briefly discussed with regard to possible mechanisms responsible for these variations and to mantle conductivity.
\end{abstract}

Key words: Geomagnetic field variation, multi-taper method, time series analysis.

\section{Introduction}

The Earth's magnetic field exhibits short and long term variations, ranging from seconds to many millions of years. Observations of the geomagnetic field allow separation of these temporal variations into two main categories, depending on their origin: external and internal, with respect to the terrestrial surface. Variations on short time scales are usually attributed to the external sources, because the conductive mantle screens out high frequency variations arising in the core. The variability of the external field variation, in fact the geomagnetic activity, is due to the response of the Earth's ionosphere and magnetosphere to the solar activity, variation of the solar wind speed and fluctuation of the interplanetary magnetic field.

In particular, the annual and semi-annual variations in geomagnetic activity have been much studied over decades (Russell and McPherron, 1973; Clúa de Gonzalez et al., 1993). Local magnetic observations show an annual variation of the geomagnetic activity, such as storms, substorms and auroras, which have been related to the annual change in solar illumination (Courtillot and LeMouël, 1988) and a variation of solar wind speed due to the change of the heliographic latitude of the Earth (Bolton, 1990; Zieger and Mursula, 1998). The annual variation was also found in the long-term occurrence of auroras (Silverman and Shapiro, 1983). Recently, Le Mouël et al. (2004) investigated the annual and semi-annual variations of geomagnetic activity, with a special interest in phase and amplitude, and also some possible mechanisms for the observed geographical variability.

In a series of papers (Malin and Isikara, 1976; Malin and

Copyright (c) The Society of Geomagnetism and Earth, Planetary and Space Sciences (SGEPSS); The Seismological Society of Japan; The Volcanological Society of Japan; The Geodetic Society of Japan; The Japanese Society for Planetary Sciences; TERRAPUB.
Winch, 1996; Malin et al., 1999) the mechanism for the annual variation of the geomagnetic field was outlined. This variation divides into two parts: an annual non-ionospheric and an independent part resulting from the seasonal modulation of Sq. The non-ionospheric term is related to the movement of the ring current towards the winter hemisphere due to the presence of the solar wind. The geometry of the annual variation is that of a $\mathrm{P}_{2}^{0}$ term.

The semi-annual variation of the geomagnetic field and that of the geomagnetic activity have extrema near the equinoxes suggesting a common origin (Malin et al., 1999; Balan et al., 2000). The semi-annual variation is due to changes in the intensity of the ring current, as a consequence of the so-called Russell-McPherron effect (Russell and McPherron, 1973), where the magnetosphere is more efficient at trapping particles at certain times (twice the year), because of the favourable geometry of the solar wind and magnetosphere. In global modelling the semi-annual variation is confined to the principal $P_{1}^{0}$ term (Banks, 1969).

In this paper, we focus on the annual and semi-annual variations observed in the monthly means of the $X$ (northward), $Y$ (eastward) and $Z$ (vertical downward) components of the geomagnetic field. The temporal variation of these components is the sum of the secular variation, the induced fields by external field variations, and geomagnetic activity variations.

The purpose of the present study is two-fold. Firstly, to conduct a detailed global time series analysis of observatory monthly means with a recently developed method for time series analysis (Ghil et al., 2002). The multi-taper method (MTM) for estimation of the spectral density function (SDF) of time series is applied to a recently up-dated monthly mean data. Secondly, to investigate the possible correlation between the regional preference of the annual and semi-annual periods in of the Earth's magnetic field components with some other geophysical properties, such 
as the global distribution of the electrical conductivity of the mantle.

\section{Data}

The present study has been performed on magnetic observatory monthly means (defined as the average over all days of the month and all sample of each day) obtained from the recently up-dated database ${ }^{1}$, from the most recent INTERMAGNET data ${ }^{2}$ and from the World Data Center Copenhagen $^{3}$. The main criterion in data selection was the length and continuity of time series of the $X, Y$ and $Z$ components. We examined observatory data within the period from 1960 to 2002 and restricted the analysis to continuous time series (no gaps allowed) of at least eight years length. Following this initial selection, all time series were checked for consistency between the annual means computed from these monthly means and those sent by the observatories to the World Data Center Edinburgh ${ }^{4}$, in order to derive reliable results.

For the present analysis, the data of 154 observatories have been analysed. Let us emphasise that no assumption was made on the secular variation of the magnetic field measurements, and no spectral filtering was applied to time series.

\section{Data Analysis}

A variety of spectral analysis techniques have been widely employed in the analysis of geophysical processes (Brillinger, 1981). More sophisticated methods have recently been developed which make more realistic assumptions on the irregular oscillatory behaviour (i.e., noise) expected in climate signals. Among these techniques, the multi-taper method (MTM) for estimating the spectral density function (SDF) of time series is one of the most promising.

\subsection{The multi-taper method}

The MTM makes use of a multiple orthogonal data taper to describe structures in time series that are modulated in frequency and amplitude. This method provides a spectral estimate with an optimal trade-off between spectral resolution and variance.

Conversely, the standard approach has been to multiply a time series by a data taper (data window such as Hanning, Blackman, Parzen, etc.) which arises when performing a discrete Fourier transform (DFT) to reduce the spectral leakage (see Percival and Walden, 1993 for details on tapering). The use of a single taper leads to large variances of the estimates of the SDF.

Thomson (1982) introduced the use of multiple orthogonal tapers to avoid this problem and to minimise the spectral leakage. The optimal tapers are defined as the eigenvectors of a minimisation problem (Slepian, 1978; Thomson, 1982).

In this study, we closely follow the procedure of Thomson (1982) and Mann and Lees (1996). For a given discrete time series $F(n)$ representing the geomagnetic obser-

\footnotetext{
${ }^{1}$ (http://www.ipgp.jussieu.fr/rech/mag/)

${ }^{2}$ (http://www.intermagnet.org/myservlet/imotbl_e.jsp)

3 (http://web.dmi.dk/projects/wdcc1/master.html)

${ }^{4}$ (http://www.geomag.bgs.ac.uk/gifs/annual_means.shtml)
}

vatory monthly means, we determine a group of independent $k, k=1, \ldots, K$ spectral estimates $Y_{k}(f)$

$$
Y_{k}(f)=\sum_{n=1}^{N} a_{k}(n) F(n) e^{i 2 \pi f n \Delta t},
$$

where $N$ is the number of data, $\Delta t$ the sampling interval and $a_{k}(n)$ is the $k$-th member of a set of $K$ orthogonal Slepian tapers (see below). From Eq. (1), the multi-taper power spectrum is constructed as

$$
S(f)=\frac{\sum_{k=1}^{K} \lambda_{k}\left|Y_{k}(f)\right|^{2}}{\sum_{k=1}^{K} \lambda_{k}} .
$$

$\lambda_{k}$ measures the fractional leakage associated with the $k^{t h}$ data taper (see Percival and Walden (1993) for details).

The choice of $K$ represents a trade off between the variance of the spectral estimate and the spectral resolution. The Slepian tapers are determined by a variation condition to minimise the spectral leakage outside the band $\pm \frac{p}{N \Delta t}$ about a given frequency $f$. It follows that the spectral resolution is given by

$$
f_{R}=\frac{2 p}{N \Delta t} .
$$

Only the first $K=2 p-1$ tapers are usefully resistant to spectral leakage (Thomson, 1982; Mann and Lees, 1996). Shorter time series require smaller values of $p$ to achieve the desired frequency resolution. In this study, we use $p=1$ for short time series, less than 20 years, and $p=2$ for those longer than 20 years, with $K=2 p-1$ the number of tapers.

\subsection{Statistical confidence}

Discriminating statistics are essential in geophysical studies, where the nature of the temporal variation is complex. We have utilised a pure line test as given by Mann and Lees (1996) to verify the significance of spectral features against the null hypothesis of a red noise background. Contrary to a white noise process, with no correlation between single observations, red noise contains some long term correlations, such as a linear trend. Therefore the red noise hypothesis is a reasonable description of the slowly varying geomagnetic continuum, e.g. secular variation. Significance levels are computed from the quantiles of a chisquared distribution, assuming that the spectrum has degrees of freedom $v \approx 2 K$. For our analysis we disregard spectral features below $99 \%$ significance.

\section{Results and Discussion}

Figure 1 shows spectra for $X, Y, Z$ components in geodetic coordinates computed for an observatory. These plots clearly indicate the existence of the annual and semi-annual variations. For some observatories (e.g., GDH) the two peaks are seen in all three components whereas for some others (e.g., HER) these peaks are more clear in one of the horizontal components. Since the behaviour of the peaks differs component to component and at observatories with similar latitude but different longitude, we decided to apply the same analysis to the field components transformed into the geomagnetic coordinates using the technique by Langel (1984). Any existing longitudinal dependence in activity 
I. WARDINSKI AND M. MANDEA: GEOMAGNETIC FIELD VARIATIONS

787
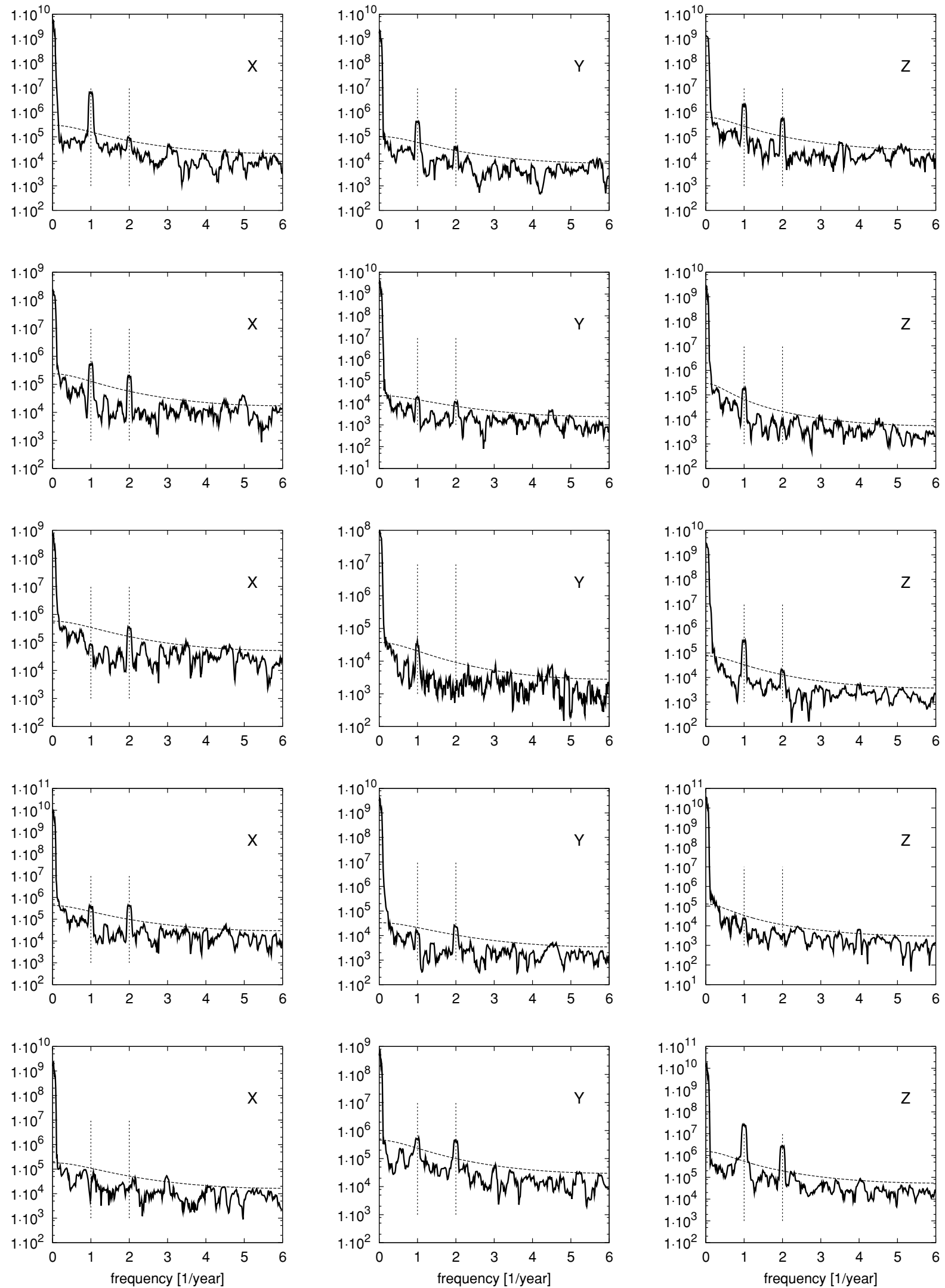

Fig. 1. Spectra for $X, Y, Z$ components derived in geodetic coordinates for the representative observatories from North (top) to South (bottom) (GDH, NGK, GUA, HER, SBA). The vertical dashed lines indicate the frequencies corresponding to the annual and semi-annual variations. The dashed line gives the $99 \%$ significance level. 

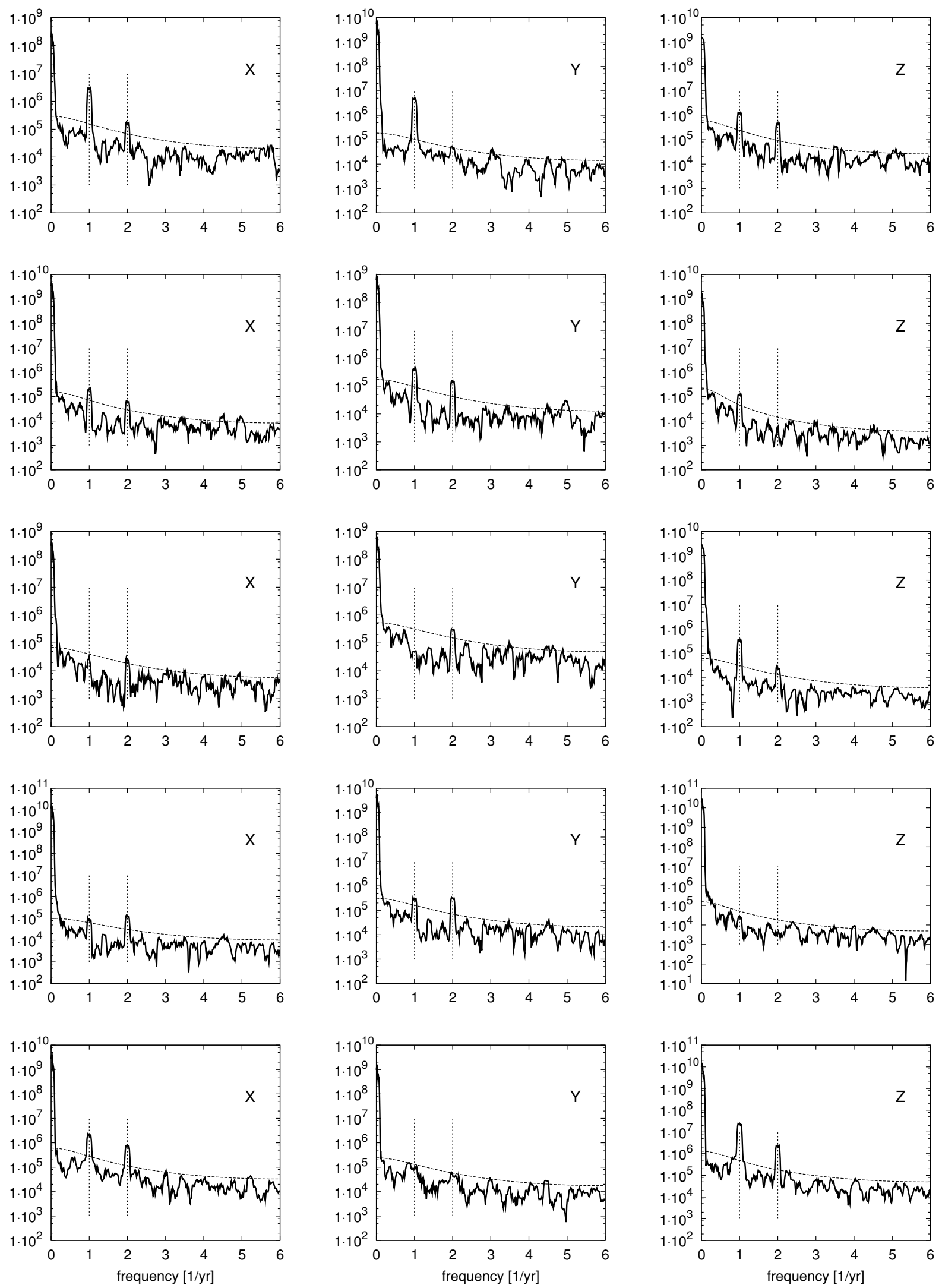

Fig. 2. Spectra for the same sites as in Fig. 1, but here the field components are computed in the geomagnetic coordinates. 


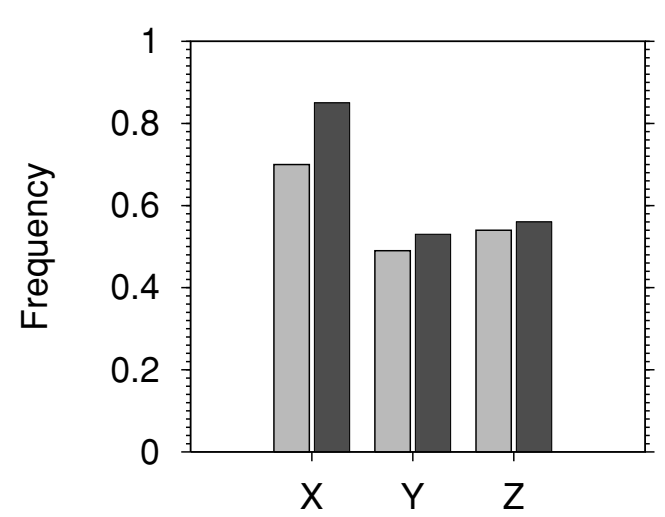

Fig. 3. Histograms of the number of observatories with significant annual and semi-annual peaks in the $X, Y$ and $Z$ components in the geodetic coordinate system. Light gray represent semi-annual and dark gray the annual variation. The vertical axes is normalised by the total number of observatories.

variations of the horizontal components due to the tilt between geodetic frame and the source field geometries of the annual and semi-annual variation should disappear by this transformation. Results are shown in Fig. 2. In these plots, the two signals are evident. The transformation of the field components from geodetic to geomagnetic coordinates affects the horizontal components significantly, and therefore their spectra. This assessment is even clearer in histograms in Figs. 3 and 4. These histograms are obtained by considering the number of observatories with significant peaks of the annual and semi-annual signals for each component. The histograms plotted are normalised by the total number of observatories. Figures 3 and 4 depict the histograms in the geodetic and geomagnetic reference frames, respectively. The transformation has clearly enhanced the peak occurrences in the $Y$ component, where the $Z$ component remains unchanged.

Figure 5 shows the annual and semi-annual variations in the vertical downward field component in geomagnetic reference frame. The heterogeneous global distribution of the annual and semi-annual signals appears striking. We consider that the heterogeneous distribution of the annual and semi-annual signals in the vertical component is an expression of the conductivity of the lower mantle, as we discuss below.

Further, in North America and some large part of the Asian region, the semi-annual variation is predominant, while in Europe the annual variation is. The situation is less clear for the southern hemisphere, where we are lacking a good observatory distribution. The annual variation is mainly present in South America, and the semi-annual variation in the southern Indian Ocean and Australia. This regional preference of the annual and semi-annual variations for the vertical downward component gives a clue in correlating them with the electrical conductivity of the mantle.

In order to investigate the possible correlation between the observed spatial distribution of the semi-annual and annual peaks, we considered a given 1-D spherical integrated conductivity model of the mantle (1). This global model is derived by expanding the monthly values into spherical harmonics and using the spherical induction code by Tarits

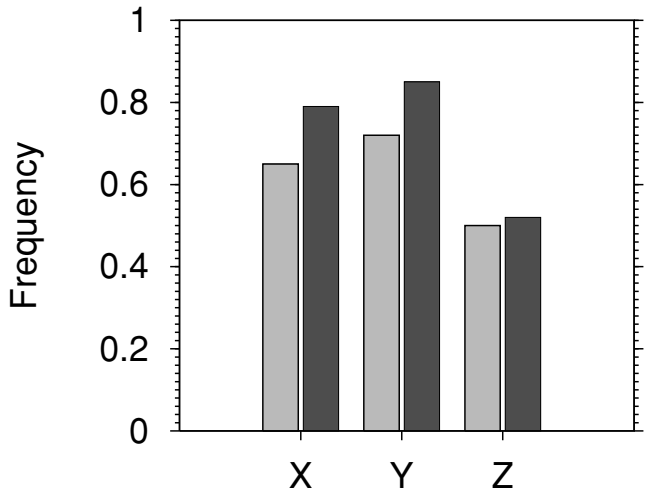

Fig. 4. Histograms of the number of observatories with significant annual and semi-annual peaks in the $X, Y$ and $Z$ components in the geomagnetic coordinate system. Light gray represent semi-annual and dark gray the annual variation. The vertical axes is normalised by the total number of observatories.

Table 1. A three layer mantle conductivity model.

\begin{tabular}{lcc}
\hline & Depth $(\mathrm{km})$ & Conductivity $(\mathrm{S} / \mathrm{m})$ \\
\hline upper mantle & $0-400$ & $0.008-0.017$ \\
mid mantle & $660-1000$ & $0.09-0.2$ \\
lower mantle & $1400-1900$ & $1.3-1.4$ \\
\hline
\end{tabular}

(1994) and Grammatika and Tarits (2002). The semi-annual field and the annual field were modelled using a $\mathrm{P}_{1}^{0}$ source field and a $\mathrm{P}_{2}^{0}$ source field, respectively. By definition the energy of a source field of degree $l$ and order $m=0$ is

$$
E=l \times q(l)^{2}
$$

where $q$ is the Gauss coefficient of the external field with degree $l$. Using a comparable source energy for both fields $(q(1) / q(2)=\sqrt{2})$, the vertical components at a geomagnetic colatitude $\left(40^{\circ}\right)$, representative for an European and an American observatory were obtained for a three layer model (Table 1, Tarits, 2004 priv. comm.). Figure 6 shows the ratios of the vertical field component of semi-annual and annual signal for the three layers with respect to the integrated conductivity. We found the semi-annual field is larger than the annual field for a lower mantle more resistive than average (see red curve of Fig. 6). While a more conductive upper mantle than average leads to a larger annual field than the semi-annual (see blue curve of Fig. 6). This analysis suggests that the different distribution of annual and semiannual field values between Europe and North America to be the result of a enhanced mantle conductivity beneath Europe than beneath North America.

\section{Conclusion}

In this paper, we analysed monthly means provided by 154 magnetic observatories for the period 1960 to 2002 in order to highlight the annual and semi-annual variations. The mechanisms causing these variations are complicated and still an open issue. The semi-annual peak is clearly better detected in the horizontal components for the observatories situated in the equatorial region. This is related to Russell-McPherron effect (Russell and McPherron, 1973) 


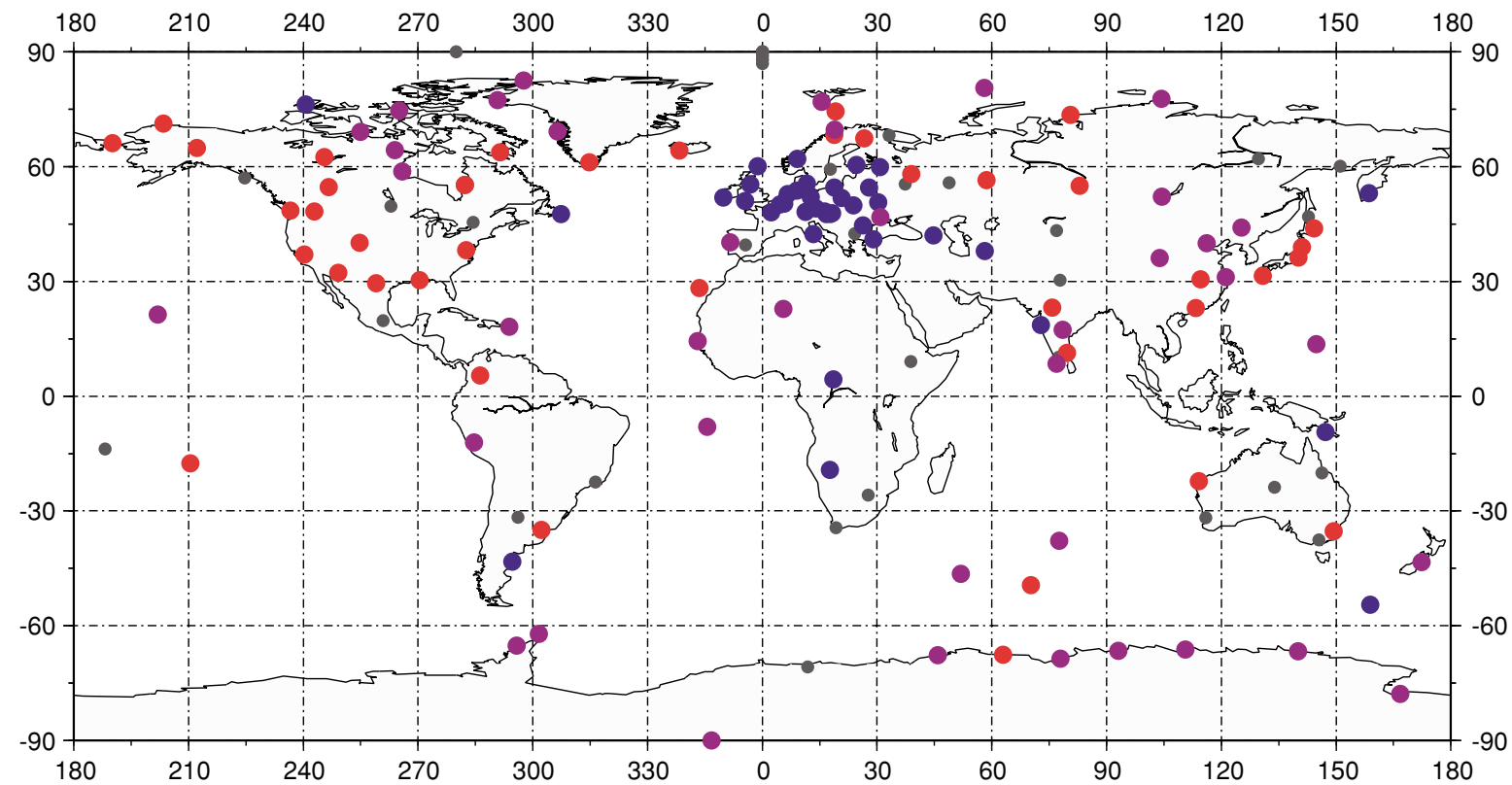

Fig. 5. Global chart of the annual and semi-annual signals in the vertical component of the observatories (geodetic frame). Red circles indicates the appearance of the semi-annual signal alone, blue circles only the annual signal, purple circles both signals and black circles no significant contribution of annual and semi-annual signal in the vertical component.

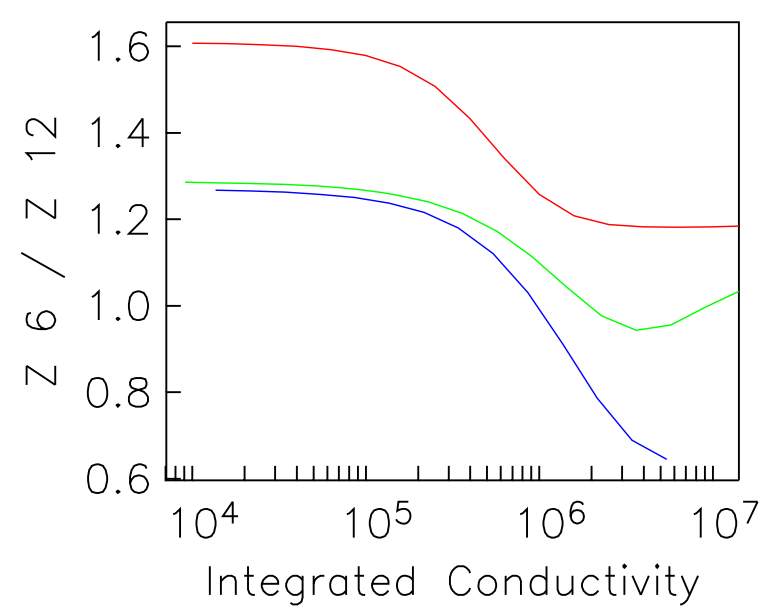

Fig. 6. This figure shows the amplitude ratios of the vertical component of the semi-annual and annual signal with respect to integrated conductivity. The curves represents these ratios for the three layers given in Table 1, red curve lower mantle, green curve mid mantle and blue curve the upper mantle.

due to the favourable geometry between the solar wind and magnetosphere. While this effect mainly explains periodicity in geomagnetic activity, it also affects the intensity of the ever-present ring current. For observatories in midand high-latitudes both peaks are well detected in horizontal components.

In this study we also showed the existence of a possible correlation of the annual variation in Europe and the semiannual variation in North America with the mantle conductivity of the two regions. Certainly, an analysis of these variations based on a decomposition of geomagnetic potential using spherical harmonics, as done by McLeod (1994) and Olsen (1999) will bring new insights into the mantle conductivity distribution at a global scale. Therefore a time- dependent description of the internal and external field is needed and will be a topic for future study.

Acknowledgments. We would like to record our gratitude to Pascal Tarits and Heather McCreadie for very helpful hints and discussions and Micheal E. Mann providing the source code for the MTM and discussing related topics. Susan Macmillan and an anonymous referee are thanked for their meticulous reviews, which have improved the quality of the paper. IW was funded during his staying at IPG Paris by the Marie-Curie fellowship (HPMT-CT-2001-00229) of the European Union.

\section{References}

Balan, N., Y. Otsuka, G. J. Bailey, S. Fukao, and M. A. Abdu, Annual variations of the ionosphere: A review based on the MU radar observations, Adv. Space Res., 25, 153-162, 2000.

Banks, R. J., Geomagnetic variations and the electrically conductivity of the upper mantle, Geophys. J. R. Astron. Soc., 17, 457-487, 1969.

Bolton, S., One year variation in the near earth solar wind ion density and bulk flow velocity, Geophys. Res. Lett., 17, 37-40, 1990.

Brillinger, R. D., Time Series: Data Analysis and Theory, Holden-Day, San Francisco, USA, 1981.

Clúa de Gonzalez, A. L., W. D. Gonzalez, S. L. G. Dutra, and B. T. Tsurutani, Periodic variation in the geomagnetic activity: A study based on the ap index, J. Geophys. Res., 98, 9215-9232, 1993.

Courtillot, V. and J.-L. Le Mouël, Time variations of the Earth's magnetic field: from daily to secular, Ann. Rev. Earth Planet. Sci, 16, 389-476, 1988

Ghil, M., M. R. Allen, M. D. Dettinger, K. Ide, D. Kondrashov, M. E. Mann, A. W. Robertson, A. Saunders, Y. Tian, F. Varadi, and P. Yiou, Advanced spectral methods for climatic time series, Rev. of Geophys., 40, 1-41, 2002.

Grammatika, N. and P. Tarits, Contribution at satellite altitude of electromagnetically induced anomalies from a 3-D heterogeneously conducting Earth, Geophys. J. Int., 151, 913-923, 2002.

Langel, R. A., The main geomagnetic field, Geomagnetism, edited by J. Jacobs, Academic Press, 1984.

Le Mouël, J. L., E. Blanter, A. Chulliat, and M. Shnirman, On the semiannual and annual variations of geomagnetic activity and components, Ann. Geophys., 22, 3583-3588, 2004.

Malin, S. R. C. and A. M. Isikara, Annual variation of the geomagnetic 
field, Geophys. J. R. astr. Soc., 47, 445-457, 1976.

Malin, S. R. C. and D. E. Winch, Annual variation of the geomagnetic field, Geophys. J. Int., 124, 170-174, 1996.

Malin, S. R. C., D. E. Winch, and A. M. Isikara, Semi-annual variation of the geomagnetic field, Earth Planets Space, 51, 321-328, 1999.

Mann, E. M. and J. M. Lees, Robust estimation of background noise and signal detection in climatic time series, Clim. Change, 33, 409-445, 1996.

McLeod, M. G., Magnetospheric and ionospheric signals in magnetic observatory monthly means: Electrical conductivity of the deep mantle, $J$. Geophys. Res., 99, 13,577-13,590, 1994.

Olsen, N., Long-period (30 days-1 year) electromagnetic sounding and the electrical conductivity of the lower mantle beneath Europe, Geophys. $J$. Int., 138, 179-187, 1999.

Percival, D. B. and A. T. Walden, Spectral Analysis for Physical Applications. Multi-Taper and Conventional Univariate Techniques, Cambridge University Press, Cambridge, UK, 1993.
Russell, C. T. and R. L. McPherron, Semiannual variation of geomagnetic activity, J. Geophys. Res., 78, 92-108, 1973.

Silverman, S. M. and R. Shapiro, Power spectral analysis of auroral occurrence frequency, J. Geophys. Res., 88, 6310-6316, 1983.

Slepian, S., Prolate spheroidal wave functions, Fourier analysis and uncertainty, V, the discrete case, Bell. Syst. Tech. J., 57, 1371-1430, 1978.

Tarits, P., Electromagnetic studies of global geodynamical processes, Surv. Geophys., 15, 209-238, 1994.

Thomson, J. D., Spectrum estimation and harmonic analysis, IEEE Proc., 70, 1055-1096, 1982.

Zieger, B. and K. Mursula, Annual variation in near-earth solar wind speed: Evidence for persistent north-south asymmetry related to solar magnetic polarity, Geophys. Res. Lett., 25, 841-844, 1998.

I. Wardinski (e-mail: ingo@gfz-potsdam.de) and M. Mandea 